\title{
Prophylactic low dose heparin anticoagulant therapy in patients with spinal cord injuries: a retrospective study
}

\author{
J R Kulkarni FRCS, ${ }^{1}$ A A Burt FRCP, ${ }^{2}$ A T Tromans FRCS, P D L Constable ${ }^{3}$ \\ ${ }^{1}$ Consultant in Rehabilitation, Withington Hospital, Cavendish Road, Manchester M2O \\ 8LR; ${ }^{2}$ Spinal Injury Unit, Pinderfields General Hospital, Wakefield; ${ }^{3}$ Department of \\ Statistics, University of Leeds, Leeds, England.
}

\begin{abstract}
A retrospective study was performed of 100 consecutive acute traumatic spinal injury patients with neurological deficit admitted to the Yorkshire regional spinal injuries centre prior to May 1990. Ninety-seven of these patients received prophylactic low dose subcutaneous heparin and 3 patients were excluded because of noted complications. Twenty-six patients developed thromboembolic complications. Apart from the fact that acute spinal cord injury patients are considered to be in an hypercoaguable state, the occurrence of deep vein thrombosis/pulmonary embolism (DVT/PE) in our study, even though these patients were on prophylactic low dose subcutaneous heparin, was related to delay in transfers, operative intervention, level of spinal cord injury and was possibly due to loss of some amount of heparin solution from the prefilled syringe during removal of air bubbles prior to the subcutaneous heparin injection.
\end{abstract}

Key words: acute spinal cord injury; deep vein thrombosis; pulmonary embolism; prophylactic heparin.

\section{Introduction}

Deep vein thrombosis (DVT) and pulmonary embolism (PE) are serious complications occurring predominantly during the first 3 months after acute spinal cord injury (SCI). ${ }^{1-3}$

The incidence of DVT varied from up to $21 \%$ by clinical and radiological diagnosis, $61 \%$ using impedance plethysmography and constrast venography, to $100 \%$ as determined by 125 I Fibrinogen uptake. ${ }^{4}$ The results of prophylactic anticoagulation for the duration of the high risk period have been described in reports from numerous spinal injury centres.

This paper reports on the prevalence of thromboembolic complications in 100 consecutive patients who were on prophylactic low dose subcutaneous heparin anticoagulation.

\section{Methods}

One hundred consecutive patients (80 males and 20 females) admitted to the Yorkshire regional spinal injury centre with acute traumatic spinal cord injury with neurological deficit prior to May 1990 were included in this study. There were 57 cervical, 34 thoracic, and 9 lumbar lesions.

Eight patients were admitted directly from Pinderfields General Hospital accident and emergency department, 87 patients were transferred from 18 other district health authorities and 5 patients were transferred from abroad (Spain 2; France 1; Pakistan 1; Thailand 1). Three out of these 5 transfers from abroad had sacral pressure sores on admission.

Ninety-seven out of the 100 patients were treated on arrival at the spinal injury centre with prophylactic low dose heparin (heparin sodium, mucous). Five thousand units were given subcutaneously 8 hourly in the anterior aspect of the thigh via a prefilled disposable syringe. No patient had been started on anticoagulants prior to admission to the spinal injury centre. Three patients were not started on the low dose heparin because of noted contraindications such as 
head injury, liver and renal contusions and haemothorax. The standard regime was to maintain subcutaneous heparin until 12 weeks from the date of admission, but if the bedrest extended to more than this duration, then the low dose heparin was continued for the length of the bedrest plus one week.

Diagnosis was made on clinical grounds with a high index of suspicion. We realised that on this basis we would not be exact in estimating the total number of thromboembolic complications. We were however concentrating on issues of everyday clinical management and therefore used our normal clinical and investigative procedures. This technique has been used successfully in the past. $^{5}$

In patients developing either DVT or PE, full heparinisation was instituted $(40,000-$ 60,000 units intravenously via a heparin syringe pump per 24 hours) and these patients were also started on a loading dose of warfarin, on which they were subsequently maintained for approximately 6 months.

Delay was defined as delay when admissions to the spinal injuries centre were delayed by more than 24 hours, from the time of the injury to the time of transfer to the spinal injuries centre.

\section{Results}

Twenty-six out of the 97 patients started on prophylactic low dose subcutaneous heparin were noted to have thromboembolic complications (DVT/PE/DVT + PE). The prevalence of thromboembolic complications (DVT/PE) in patients on prophylactic low dose subcutaneous heparin is shown in Table I. The levels of spinal cord injury in the thromboembolic and non thromboembolic group is shown in Table II.

In 33 out of the 97 consecutive patients, there was a delay in transfer to the regional spinal injuries centre ranging from 2 days to 87 days. Forty-two per cent (14 out of 33) of the delayed arrivals ( $>24$ hours) had thromboembolic complications. The delay group was subdivided into: no delay/1-7 days $/ 8-14$ days $/ 8-14$ days $/>15$ days.

Overall, 47 out of 97 patients in the study
Table I Prevalence of thromboembolic complications: 26 out of 97 patients

\begin{aligned} & \hline Patients complication \\ & \hline 14 Deep vein thrombosis (DVT) in left leg \\ & 3 DVT in right leg \\ & 7 Pulmonary embolism (PE) \\ & 2 DVT + PE \\ & \hline\end{aligned}

Table II Level of spinal cord injury

\begin{tabular}{lrcc}
\hline Patients & \multicolumn{3}{c}{ Spinal cord injury } \\
\hline $\begin{array}{l}\text { Patients with } \\
\text { thromboembolic }\end{array}$ & Cervical & Thoracic & Lumbar \\
complications & & & \\
$\quad$ DVT & 9 & 3 & 4 \\
$\quad$ PE & 0 & 7 & 1 \\
$\quad$ DVT + PE & 0 & 2 & 0 \\
Patients with no & 48 & 19 & 4 \\
thromboembolic & & & \\
complications & & & \\
\hline
\end{tabular}

had operative intervention. In the thromboembolic group $84 \%$ of the patients had operative intervention, whilst in the non thromboembolic group, $35 \%$ had operative intervention.

Patients were then cross classified as those with DVT, those with PE or those with both DVT + PE on the one hand and delay (days), operative intervention (yes/ no), and level of spinal cord injury (cervical/ thoracic/lumbar) on the other. The significance levels achieved by Chi-squared tests ( $p$ values) of the hypothesis if no association was assumed are shown in Table III.

It is thus evident that patients having operative intervention had a higher chance of DVT, and those having operative intervention and thoracic injuries had a higher chance of PE. It was noted that a higher proportion of those delayed prior to transfer to the spinal injuries unit had DVT or PE but this was not statistically significant.

Logistic regression which allows closer examination of the combined effect of the independent factors (delay, operative intervention, level of injury) on the chances of the dependent variable occurrence (DVT, 
DVT + PE) was performed. Only significant terms were retained in the following model:

$$
\begin{aligned}
& \text { Probability }(\text { DVT })= \\
& \frac{\mathrm{e}^{\mathrm{bx}}}{1+\mathrm{e}^{\mathrm{bx}}} \text { where } \mathrm{bx}=\begin{array}{l}
-2.75+2.09 \mathrm{X} 2 \\
(0.60)(0.67)
\end{array} \\
& \text { Probability }(D V T / P E)=\frac{e^{b x}}{1+e^{b x}}
\end{aligned}
$$

where $b x=$

$$
\begin{gathered}
-2.17+2.37 \mathrm{X} 2-1.00 \times 31+0.31 \times 32 \\
(0.55)(0.62) \quad(0.39) \quad(0.40)
\end{gathered}
$$

where $\mathrm{X} 2=1$ if operative intervention was required 0 otherwise

$$
\begin{aligned}
\mathrm{X} 31= & 1 \text { cervical injury } \\
& 0 \text { thoracic injury } \\
- & 1 \text { lumbar injury } \\
\mathrm{X} 32= & 0 \text { cervical injury } \\
& 1 \text { thoracic injury } \\
- & 1 \text { lumbar injury }
\end{aligned}
$$

Hence from logistic regression the probability of DVT increases from $6 \%$ if no operative intervention is required, to $34 \%$ if is required. The estimated probability of DVT or PE increases from $4 \%$ for cervical injury patients with no operative intervention, to $71 \%$ for lumbar injury patients with operative intervention.

\section{Discussion}

The aim of prophylactic anticoagulation in patients with traumatic acute spinal cord injury is to lower the coagulability of the blood by keeping the concentration of Factor Xa below the critical level which would lead to thrombin generation and thromboembolic complications. ${ }^{6}$

The benefit and the notable decrease in mortality and morbidity with prophylactic anticoagulant therapy in patients with spinal cord injury is well established. There was no mortality in the patient population of this study. The question as to the occurrence of (26\%) thromboembolic complications even though the patients were on prophylactic low dose heparin was addressed.
Table III Significance levels ( $p$ value)

\begin{tabular}{lccc}
\hline $\begin{array}{l}\text { Thromboembolic } \\
\text { complications }\end{array}$ & Delay & $\begin{array}{c}\text { Operative } \\
\text { intervention }\end{array}$ & Level \\
\hline DVT & 0.14 & $<0.001$ & 0.21 \\
PE & 0.36 & 0.01 & $<0.001$ \\
DVT + PE & 0.06 & $<0.0001$ & 0.02 \\
\hline
\end{tabular}

Delay in transfer to spinal injury units has been addressed in the past and is best avoided. ${ }^{5,7}$

Sixty-three per cent of all traumatic spinal cord injuries were admitted directly from accident and emergency departments. The remainder fell into 3 categories: those who had multiple injuries requiring immediate emergency life saving surgery, those from abroad; and those with spinal cord injuries but with no associated injuries, who for no obvious reason were referred late. The patients were accepted from the referring accident and emergency department provided they were haemodynamically stable.

The delays in referral to spinal injuries units, must be in part related to people's understanding of the spinal injuries units' function as a rehabilitation facility and not as an acute treatment service facility.

The possibility of inadequate potency of the heparin sodium solution in the disposable syringe was checked by sending the same to the regional pharmaceutical laboratory for analysis, but these were reported to be normal.

Subcutaneous injection of low dose heparin 5000 units 8 hourly was injected in the anterior aspect of the thigh by the nursing staff. During administration of the injection it was noted that the disposable prefilled subcutaneous heparin syringes had some air bubbles in them and on trying to get rid of these air bubbles some of the heparin sodium solution was wasted. Thus a lower dose of heparin was thereby injected. The amount of heparin solution lost was at times a quarter of the total amount. There were no reported complications, local or general from the use of the subcutaneous low dose heparin injection.

Patients who were delayed prior to transfer to a spinal injuries centre and who 
subsequently had operative intervention were more at risk of developing thromboembolic complications.

$\mathrm{Kakkar}^{8}$ did report failure of low dosage heparin in prophylaxis of DVT in patients with fractured necks of femur. Watson ${ }^{3}$ advocated subcutaneous low dose heparin of 5000 units twice daily for a month and then a switchover to warfarin by the fourth week.

From our observation we feel that even a higher dose of 5000 units thrice daily could be inadequate for prophylaxis in 'at risk' patients and that due care has to be given to the administration of the injection.

Lastly, further studies are needed to elucidate the optimum regime of prophylactic low dose heparin anticoagulation in patients with acute spinal cord injuries and early referral to spinal injury unit must be encouraged.

\section{Acknowledgement}

We would like to thank Mrs secretarial assistance.

\section{References}

1 Phillips RS (1963) Incidence of deep venous thrombosis in paraplegia. Paraplegia $\mathbf{I}$ : 116.

2 Walsh JJ, Tribe LR (1965) Phlebothrombosis and pulmonary embolism in paraplegia. Paraplegia 3: 209-213.

3 Watson N (1978) Anticoagulant therapy in the prevention of venous thrombosis and pulmonary embolism in the spinal cord injury. Paraplegia 16: 265-269.

4 Todd JS, Frisbie JH, Rossier AB, Adams DF, Armenia AV, Sassahaka AA et al (1976) Deep vein thrombosis in acute spinal cord injury: a comparision of 125 fibrinogen leg scanning, impedance plethysmography and venography. Paraplegia 14: 50-57.

5 El Masri WS, Silver JR (1981) Prophylactic anticoagulant therapy in patients with spinal cord injury. Paraplegia 19: 334-342.

6 Perkash A (1980) Experience with the management of deep vein thrombosis in patients with spinal cord injury. Paraplegia 18: 2-14.

7 Carvell J, Grundy D (1989) Patients with spinal injuries. Early transfer to a specialist centre. $\mathrm{Br} \mathrm{Med} J$ 299: 1353-1354.

8 Kakkar VV, Corrigan T, Spindler J, Fossard DP, Flute PT, Crellin RQ, Wessler S, Yin ET (1972) Efficacy of low dose heparin in prevention of DVT after major surgery. Lancet, ii: 101. 